\title{
Editorial to a feature issue on advances in behavioural research on supported decision processes
}

\author{
Gilberto Montibeller $^{1} \cdot$ Jyrki Wallenius ${ }^{2}$
}

Received: 7 April 2018 / Accepted: 10 April 2018 / Published online: 24 April 2018

(C) Springer-Verlag GmbH Germany, part of Springer Nature and EURO - The Association of European Operational Research Societies 2018

The previous Editor-in-Chief, Ahti Salo, asked us to co-edit a Feature Issue or a Special Issue about behavioural research on supported decision processes. We gladly accepted. We both have conducted a significant amount of research related to behavioural decision-making. We had a Call for Papers in the winter of 2016. Despite our efforts, we only received four submissions. Out of the four submissions, we accepted two: a paper by Matthew Welsh and Steve Begg and a paper by Ian Durbach and Gilberto Montibeller. Gilberto processed two of the submitted papers, and Jyrki one plus the Durbach and Montibeller paper.

The Welsh and Begg paper discusses the so-called 'More-Or-Less-Elicitation', an elicitation process to mitigate or eliminate well-known biases in human judgment, such as over confidence and anchoring. Their idea relies on several key insights, such as 'humans are better at relative than absolute judgments' and 'repeated estimates should be averaged to produce a better estimate'. The Durbach and Montibeller paper examines the impact of negative, rare, and extreme events on judgmental forecasts. Their paper is based on a behavioural experiment. The results support the view that decision-makers under weigh rare and extreme events rather than over weigh them.

We do not think that the low number of submissions reflects a lack of interest in behavioural issues in decision-making, but instead on the methodological rigor that we requested in our Call for Papers. Indeed, we see many signs that behavioural decision-making, and behavioural OR in general, is receiving increasing attention by the scientific community. It is interesting to note that the field is not new. Ward Edwards, Herbert Simon, Daniel Kahneman, and Amos Tversky all have pioneered

Jyrki Wallenius

jyrki.wallenius@aalto.fi

1 Loughborough University, Loughborough, UK

2 Aalto University, Espoo, Finland 
behavioural decision research. In fact, three of the Economics Nobel Prize recipients have been behavioural decision theorists: Simon, Kahneman, and most recently Thaler. Yet, for many decades, their work did not have a large impact on mainstream Management Science/Operations Research, which developed separately from behavioural decision theory. Alberto Franco and Raimo P. Hämäläinen established the Behavioural OR Working Group a couple of years ago. They have also been organizing highly popular streams at recent EURO and IFORS Conferences, helping to further develop the behavioural OR field. We hope that this feature issue helps in further promoting the relevance of behavioural issues in decision processes.

Gilberto Montibeller

Loughborough University, UK

Jyrki Wallenius

Aalto University, Finland 